\title{
Cognitive Based Electric Power Management System
}

\author{
Tahir Cetin Akinci and Alfredo A. Martinez-Morales
}

\begin{abstract}
An electric power network can be evolved into smart grids, which are measured by providing energy efficiency and improving the available resources. With the development of software and hardware elements, the decision-making mechanism of existing smart grids is transformed into more robust uninterrupted and economical energy management systems. In this study, a cognitive-based algorithm using dynamic energy management flexibility, storage and energy management algorithm and cloud computing architecture is proposed. Using this approach, an uninterrupted and economical energy management system can be planned. In addition, the proposed approach provides the optimization of supply and demand sides.
\end{abstract}

Index Terms-Cognitive Management, Electric Power Management, Electric Power Price Management System, Cognitive Network System.

\section{INTRODUCTION}

$\mathrm{C}$ OGNITIVE engineering is an area that provides decision support, human-automation interaction by redesigning existing knowledge in cognitive science and engineering [1]. This area is used in all risky areas from health to energy. Cognitive engineering; cognitive science, data science, data analysis methods and analytical and software-based methods are combined. For example, in control engineering applications, controllers in the role of human beings who manage semi-autonomous systems can be considered an application for cognitive systems. These semi-autonomous systems reduce the physical workload of people while reducing human-induced failures. Almost all of these applications are computer-based systems. Thus, the development of the experience of computer systems and software technology will enable the creation of more cognitive technology environments in the future [1-4].

TAHIR CETIN AKINCI, is with Department of Electrical Engineering University of Istanbul Technical University (ITU), Istanbul, Turkey, (e-mail: akincitc@itu.edu.tr). And University of California Riverside, WCGEC, Riverside, CA, USA. (tahircetin.akinci@ucr.edu)

https://orcid.org/0000-0002-4657-6617

ALFREDO A. MARTINEZ-MORALES, is with CE-CERT, University of California Riverside (UCR), Riverside, CA, USA, (e-mail: alfmart@ece.ucr.edu).

https://orcid.org/0000-0003-4204-2228

Manuscript received September12, 2021; accepted January 08, 2022. DOI: 10.17694/bajece. 1060998
Cognitive Engineering methods allow you to solve natural complex problems in the workspace by giving them simple attributes. The results of the analyses obtained as a result of Cognitive Engineering methods can be put into action and can be designed [3-7].

\section{A. Cognitive Approach to Electrical Power Engineering}

Computer and software technology are also closely related to energy management systems. Intelligent systems with bidirectional communication capability, which provide the energy supply and the coordination between the demanders, are developing every day [8]. One of the most important features of the smart grid is to provide energy efficiency and management of existing resources [9]. Smart Electricity Networks are focused on providing the most economical way to ensure the continuity of energy transmission and distribution in the coordination of all the elements within the system. Energy quality, continuity and energy economy are the three most important elements of energy transmission and distribution systems. The integration of these elements ensures the flexibility of the energy systems. Energy security is another of the elements that are considered in terms of energy security both by supply and demand [8-11]. In this context, smart grid systems enable the communication of information between consumers and producers. In Figure 1, this flow in energy architecture is given [12].

This mutual communication in the intelligent network architecture helps control the supply and demand balance between producers and consumers by checking every moment of the detection and measurement components on energy power systems. Nowadays, public service companies use smart meter technology to gain access to the requested information to monitor the supply-demand balance. Besides, the supply or demand side of the energy can be measured by wired or wireless methods by developing sensor technology. The obtained information is combined and system response is prepared by using decision-making algorithms. The Smart Grid market is developing in a direction that will encourage both the customer and the manufacturer with the development of data analysis application methods. Recently, information and communication technologies are used in electrical power systems. Electrical energy management algorithms provide important solutions to power decision-making in efficient ways. Especially successful in reducing consumer electricity energy bills is achieved. 


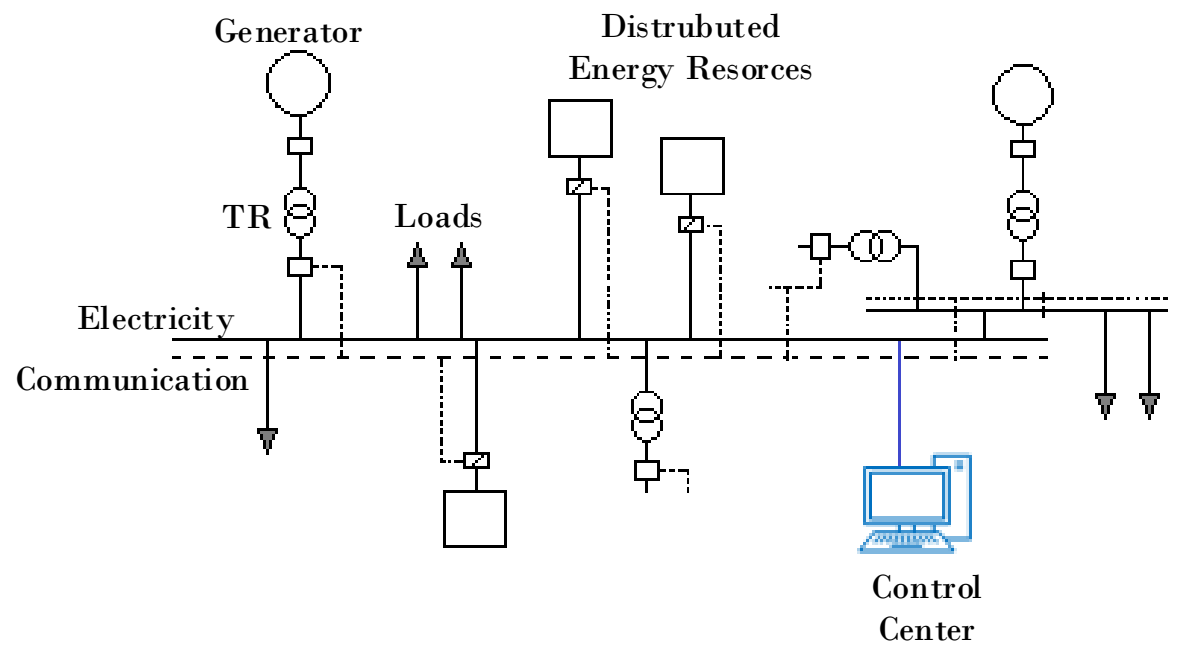

Fig.1. Architecture of a Typical Energy System

In addition, energy management, autonomy practices in controlling different power grid subsystems (energy) allow consumers to choose suppliers. In the literature, many definitions of energy management have been associated with the energy strategy [14-19]. In this sense, it would not be wrong to define and associate energy management with planning, strategy and policy. These definitions may reveal the idea of controlling the management approach in the distribution and planning of energy transmission with a Cognitive-based approach.

In this study, a cognitive-based electrical energy management system was researched.

\section{ENERGY MANAGEMENT REQUIREMENTS AND ELECTRICITY MARKETS}

The increase in the population and the development of the industry increase the demand for electrical energy. Energy providers have to provide steady, high quality, continuous and economic energy to meet the growing demands. However, the fact that energy cannot be supplied from a single source and from a single producer can lead to complex problems, including environmental, commercial and sometimes international agreements. While the demand response is required for the stability of the electricity grid operation, the energy supply side forces consumers to offer tariffs that motivate them to save energy and/or shift loads into intense periods. In addition, unbalanced energy usage demands reveal the problems of buffering and storing energy.

\section{DEMAND RESPONSE FOR ELECTRIC POWER DISTRIBUTION}

In the energy markets, the Demand Response (DR) could be considered as a stimulating opportunity for energy consumers to participate in these electricity markets. Smart grids are important for the integration of DR, both by demand and by energy supply organizations. The main role of DR in the electricity market is that it economically optimizes supplydemand balance during peak hours. Without changing the energy consumption during the high price hours, it shifts some of the high demand processes to non-peak periods and makes optimum adjustments [8,13,14].

\section{CLOUD COMPUTING}

Many authors used cloud computing in smart grid energy management. In this section, we will present some of the proposed algorithms. Cloud systems are hierarchically placed into three basic groups. These are Hybrid, Public and Private. (Figure 2). Within this group, users have the possibility to select Cloud that is suitable for data processing capacity $[8,18]$.

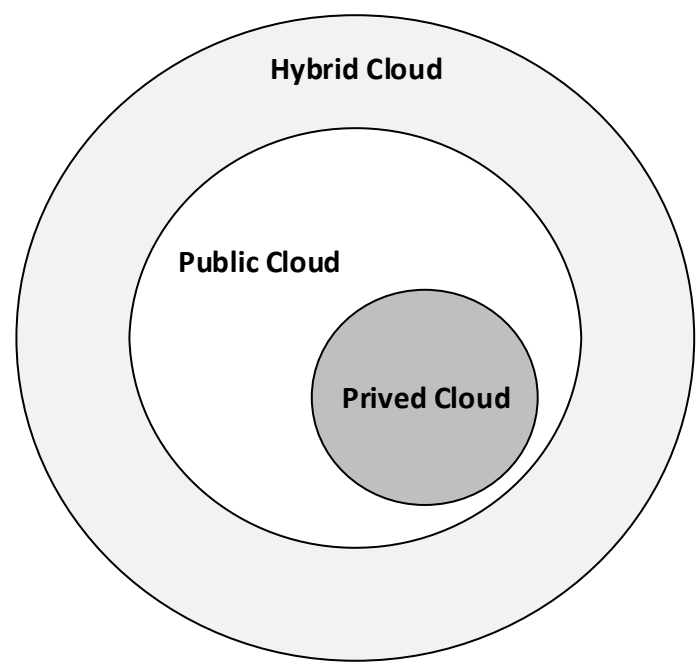

Fig.2. Cloud Systems Groups $[8,18]$.

Cloud Computing (CC) is defined as a data sharing based model. CC enables independent operating systems, data warehouses and hardware infrastructures to be shared via software via servers. It provides users with virtual machines, software, and storage physical virtual resource infrastructure. $\mathrm{CC}$ allows users to run applications without installing software 
on their own computers and have a wide range of uses [18] (Figure 3).

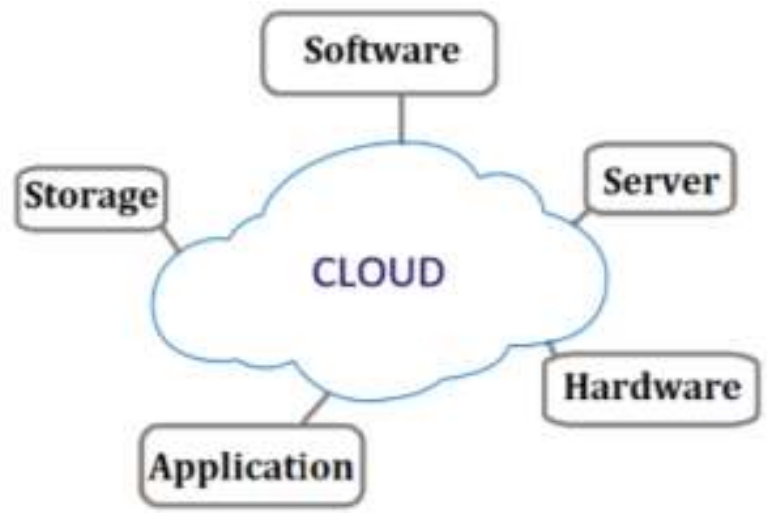

Fig. 3. General Uses of Cloud Systems

\section{A. Fog Computing}

Fog Computing is an extension or part of the Cloud network. However, it is more accessible and less extensive [21,22]. With Fog Computing, end users can carry out data processing, storage, and service between the traditional Cloud systems. Fog Computing has many advantages. These; fast, high geographic distribution, a large number of real-time interaction. In terms of quality, Fog Devices have three levels of hierarchy. This hierarchy; Cloud Level can be classified as Fog Level and Smart Devices. Fog Computing very suitable for intelligent grid applications in electrical power systems. In the networks, energy load balancing, smart meters and microgrid applications are the most common applications. It also offers practical algorithm approaches for planning and management for power systems [22].

\section{COGNITIVE SYSTEMS}

Human-based expert systems are defined as cognitive systems. Establishing a knowledge-based system by transferring human knowledge and expertise to learning systems with programming systematic is defined as Cognition. Cognitive systems engineering has recently played a key role in science and technology. Since cognitive systems are the systems that learn and think of human beings, a human-based expert enables cognition in the system through learning algorithms and logic [23]. Cognition terminology covers mental processes such as perception, memory, thought, and learning [24]. Today, cognition has very popular applications in social sciences and science. In engineering, the term Cognitive Systems finds application and is considered a set above artificial intelligence methods. The increase of cognitive-based applications in industrial and engineering systems makes the performance of the systems determine $[6,25]$.

When the cognitive decision-making mechanism is considered as the supercluster of artificial intelligence, the relationship between the concept of artificial intelligence and the cognitive system can be expressed as in Figure 4. In this sense, cognitive system engineers take part in the design of human processes, communication systems, teams and management systems that coordinate various activities within the specified processes. Cognitive System engineering will be the key engineering role in the control of many critical and complex systems in the future.

Information processing is performed by processors in electronic equipment. In fact, processors are not just hardware on computers. Many electronic systems now have a processor, which allows control with the processor. The first processor was the beginning of a new era in the hardware world, thus enabling the development of the computers we use today. However, the most important information processor is the human brain. The human brain system attempts to achieve a match between models and objects by using real-life objects or mathematical models. They are stored in the processor's memory, creating inputs and outputs to generate symbolic expressions represented by some symbols. In an immense computing system such as the human brain, the mathematical or abstract model is provided by the capability of the processing units. This structure can be explained by Cognitive Systems or Cognitive engineering [26,27].

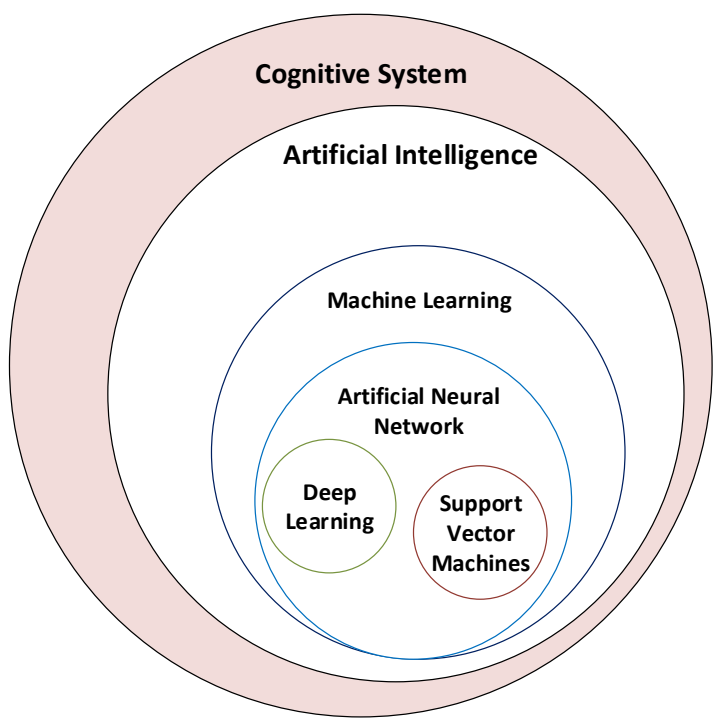

Fig.4. Relationship between Cognitive Systems and Artificial Intelligence Methods

\section{A. The framework of Cognitive Learning Systems}

Cognitive Systems (CS) is a new discipline in which brain and natural intelligence are shared by psychology, philosophy, mathematics, statistics and engineering disciplines. This discipline, covers many topics, from social psychology to industrial autonomous systems. In the field of engineering, artificial intelligence, statistics and software engineering are widely used. It is possible to explain the mathematical background representing the cognitive systems with the Cognitive Information Model (CIM). The concept of examining the four basic sections of the CIM makes it easier to explain. These; Knowledge $(K)$, Behaviour $(B)$, Experience $(E x p)$, and Skill (Skil). Learning $\left(L_{\text {earn }}\right)$ is expressed here; 
CIM model of knowledge $\left(\mathcal{L}_{\text {know }}\right)$, experience $\left(\mathcal{L}_{\text {exp }}\right)$ and skill $\left(\mathcal{L}_{\text {skil }}\right)$ is expressed by [15-17].

$\mathcal{L}_{\text {Learn }}=\left\{\mathcal{L}_{\text {know }}, \mathcal{L}_{\text {beh }}, \mathcal{L}_{\text {exp }}, \mathcal{L}_{\text {skil }}\right\}$

It can also be expressed here with Cognitive Learning $\left(\mathcal{L}_{\text {cog }}\right)$. In fact, Cognitive Learning is a form of Transitive Learning.

$\mathcal{L}_{\text {cog }} \triangleq\left\{\mathcal{L}_{\text {know }}, \mathcal{L}_{\text {beh }}, \mathcal{L}_{\text {exp }}, \mathcal{L}_{\text {skil }}\right\}$

$\mathcal{L}_{\text {cog }} \triangleq f_{\text {cog }}:(S T M) \rightarrow K(L T M)$

Perceptive Cognitive Behavioural;

$$
\begin{aligned}
& \mathcal{L}_{\text {pcogbeh }} \stackrel{\wedge}{=} \mathcal{L}_{p} \rightarrow \mathcal{L}_{\text {cog }} \rightarrow \mathcal{L}_{\text {beh }} \\
& f_{\text {pcogbeh }}: D(S B M) \rightarrow I(S T M) \rightarrow K(L T M) \rightarrow B(A B M)
\end{aligned}
$$

Cognitive Introspective;

$\mathcal{L}_{\text {cogint }} \triangleq \mathcal{L}_{\text {cog }} \rightarrow \mathcal{L}_{\text {int }}$

$f_{\text {cogint }}: I(S T M) \rightarrow K(L T M) \rightarrow I(S T M)$

Equations 3 and 4 are shown in Cognitive Models of Memory (CMM), Sensory Buffer Memory (SBF), Short Term Memory (STM), Long Term Memory (LTM) and Action Buffer Memory (ABM) [15-17]. In this sense, it can be used in the planning and pricing of the mentioned learning systems, energy management systems.

\section{CONCEPT OF COGNITIVE ENERGY MANAGEMENT SYSTEM}

Cognitive systems are topologically related to a lot of information and data. Therefore, it may be possible to explain the cognitive system model obtained by human-based expert and neural network with this procedure.
In this algorithm, logical coding and data structure is applied to train information through the neural network. As a result, this newly trained neural network plays the role of logic modelling. And such a system can be used in the electrical power systems management system. The algorithm flow chart of this system is shown in Figure 5.

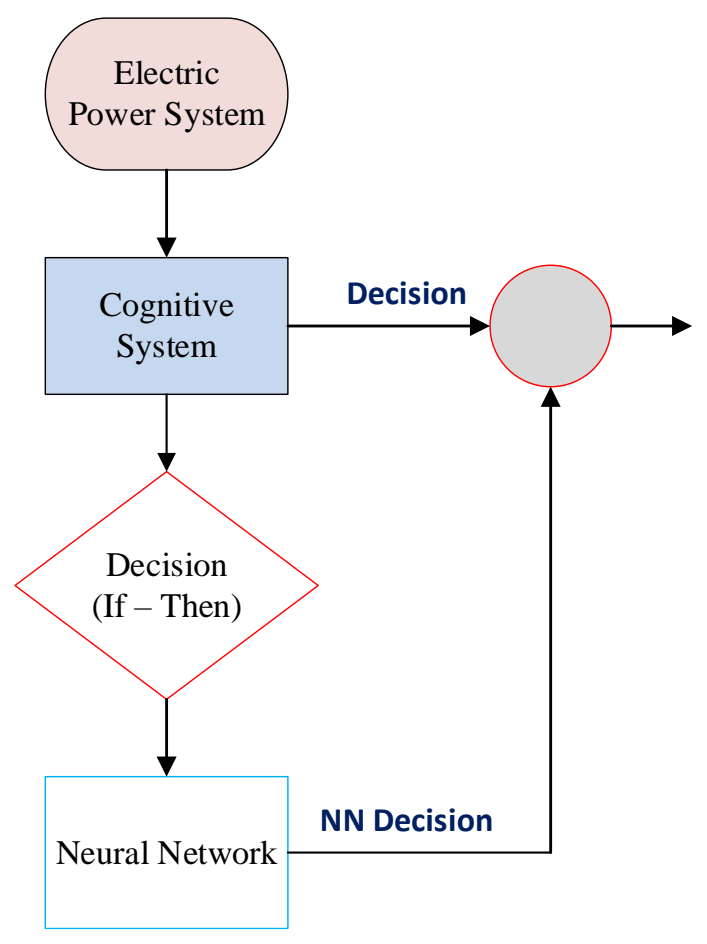

Fig. 5. Cognitive Electric Power Flowchart

As shown in the flowchart shown in Figure 5, the second block has a cognitive or human-based (expert) operator. The block with the if-then structure is associated with the neural network block for the decision.

Although the decision structure is given symbolically as a neural network, it works as a machine learning decision-maker in this block.

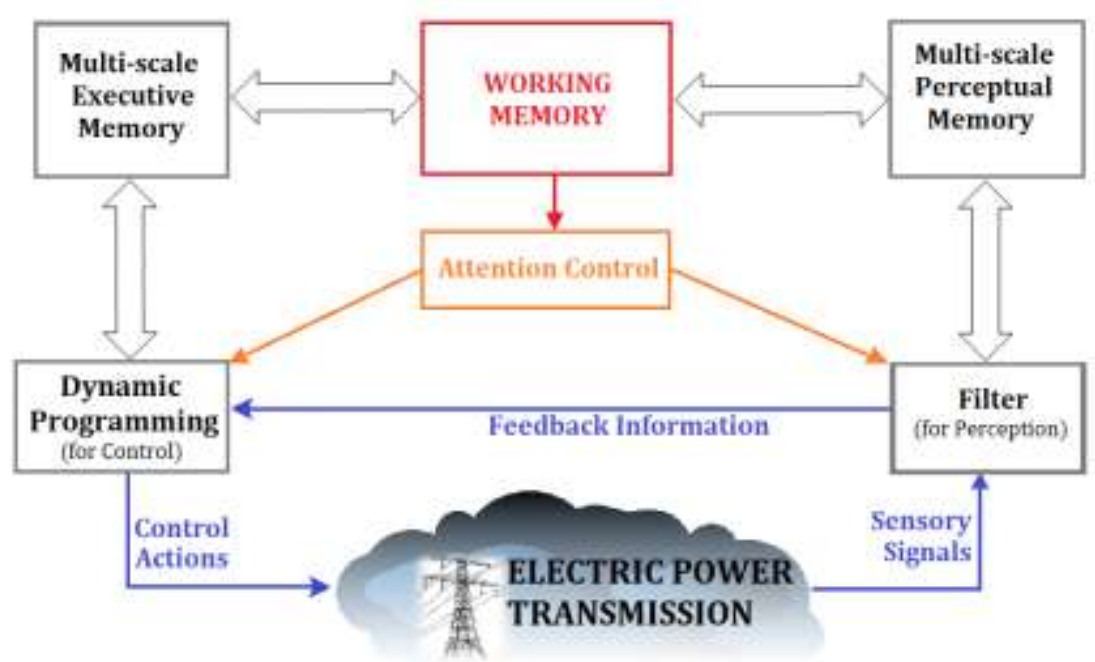

Fig. 5. Block diagram of Electric Power Transmission Management System [12]. 
The Cognitive Energy Management System (CEMS) controls and plans all stages of energy transmission from generation to end-user through the cloud. It includes hardware infrastructure as well as software and market research. Working Memory controls the operation of collecting all data. Below it is Attention Control. Dynamic Control and Perception Filters balances the system at every stage of the organization (Fig.5). The main purpose of Working Memory is to control the data on the Electric Power Transmission Line using sensors.

Block diagram of Cognitive base Electric Power Price Management System is shown in Figure 6. Here, the decision to determine the price in the energy markets is determined by the Cognitive system over the cloud.

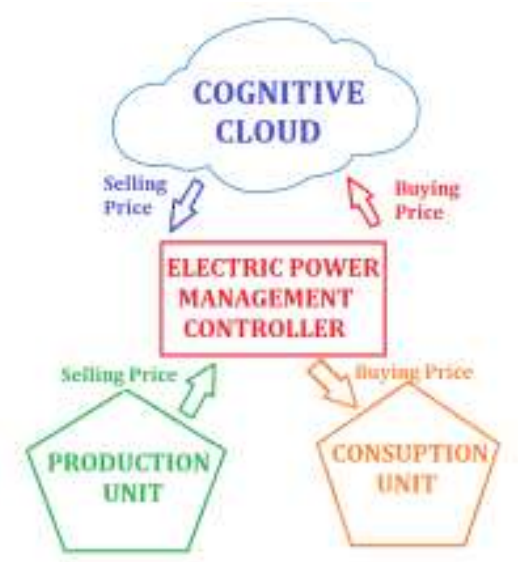

Fig. 6. Block diagram of Cognitive base Electric Power Price Management System

\section{CONCLUSION}

The integration of electric power systems is becoming more and more important. The main objective of the integration is to ensure the transmission of quality and uninterrupted energy to the users by using flexible integration strategies of energy sources. In this study, the management and planning of cognitive-based energy systems and the organization of the energy economy are examined. In addition, pricing management has been examined under the control of Electric Power Systems. Relationships between working memory and control actions are given in energy transmission lines. In this study, a cognitive-based algorithm that uses a storage and energy management algorithm and cloud computing architecture is proposed. This algorithm also has dynamic energy management flexibility.

\section{ACKNOWLEDGMENT}

This study was supported by TUBITAK (The Scientific and Technological Research Council of Turkey) 2219International Postdoctoral Research Scholarship Program, with project number 1059B192001347 in the 2020/2 application period.

The authors would like to thank TÜBİTAK for their support.

\section{REFERENCES}

[1] A.Z. Hettinger, E.M. Roth, A.M. Bisantz, "Cognitive Engineering and Health Informatics: Applications and Intersections", Journal of Biomedical Informatics, Vol.67, pp.21-33. 2017.

[2] Y. Wang, "The Theoretical Framework of Cognitive Informatics", International Journal of Cognitive Informatics and Natural Intelligence, Vol.1, No.1, 2007, pp. 1-27.

[3] V.L. Patel, T.G. Kannampallil, "Cognitive Informatics in Biomedicine and Healthcare", Journal of Biomedical Informatics, Vol.53, 2015, pp. 3-14.

[4] M.R. Endsley, R. Hoffman, D. Kaber, E. Roth, "Cognitive Engineering and Decisionmaking: an Overview and Future Course", Journal of Cognitive Engineering and Decision Making, Vol.1, No.1, 2007, pp.121.

[5] D. A. Norman and S. W. Draper, User Centered System Design: New Perspectives on Human-Computer Interaction, CRC Press, 1986.

[6] E. Hollnagel, and D.D. Woods, "Cognitive Systems Engineering: New Wine in New Bottles", International Journal of Man-Machine Studies, 1983, Vol.18, pp.583-591.

[7] E. Hollnagel, D.D. Woods, Joint Cognitive Systems: Foundations of Cognitive Systems Engineering, CRC Press, 2005, 113-155.

[8] J. Klaimi, R. Rahim-Amoud, L. Merghem-Boulahia and A. Jrad, Energy Management Algorithms in Smart Grids: State of the Art and Emerging Trends, International Journal of Artificial Intelligence and Applications (IJAIA), Vol.7, No.4, 2016, pp.25-45.

[9] G. Yuanxiong, M. Pan, Y. Fang, "Optimal Power Management of Residential Customers in the Smart Grid", IEEE Transactions on Parallel and Distributed Systems, Vol.23, No.9, 2012, pp.1593-1606.

[10] R. Belkacemi, A. Bababola, Experimental Implementation of MultiAgent System Algorithm for Distributed Restoration of a Smart Grid System, IEEE SOUTHEASTCON, 2014.

[11] T. Nagata, Y. Ueda, M. Utatani, A multi-agent approach to smart grid energy management, 10th International Power \& Energy Conference (IPEC), pp.327-331, Dec 2012.

[12] Y. Xue, S. Sirouspour, and A. Emadi, Towards Cognitive Energy Management System of Microgrid in Enabling Transportation Electrification, 2012 IEEE Transportation Electrification Conference and Expo (ITEC).

[13] B. Bitzer, and E.S. Gebretsadik, Cloud Computing Framework for Smart Grid Applications, 48th International Universities' Power Engineering Conference (UPEC) 2013 48th International Universities', 2013, pp.1-5.

[14] S. Pierluigi, "Demand response and Smart Grids-A Survey", Renewable and Sustainable Energy Reviews, Vol.30, 2014, pp.461478.

[15] Y. Wang, Using Process Algebra to Describe Human and Software System Behaviors. Brain and Mind:, A Transdisciplinary Journal of Neuroscience and Neurophilosophy, Vol.4, No.2, 2003, pp.199-213.

[16] Y. Wang, The Theoretical Framework and Cognitive Process of Learning, Proc. $6^{\text {th }}$ IEEE International Conference on Cognitive Informatics (ICCI'07), 2007, pp.470-479.

[17] L. Ogiela, "Towards Cognitive Economy", Soft Computing, 2014. Vol.18, No.9, pp.1675-1683.

[18] D.C. Wyld, Cloud Computing and the Public Sector Around the World, International Journal of Web \& Semantic Technology, Vol.1, No.1, 2010, pp.1-20,

[19] H.F. Atlam, R.J. Walters and G.B. Wills, "Fog Computing and the Internet of Things: A Review", Big Data Cognitive Computing, Vol.2, No.10, 2018, pp.2-18.

[20] O. Aizpurúa, R. Galán, A. Jiménez, A New Cognitive-Based Massive Alarm Management System in Electrical Power Administration, Proceedings of the 7th International Caribbean Conference on Devices, Circuits and Systems, Mexico, Apr. 28-30, 2008.

[21] E.M. Rantanen, J.A. Winkle, T.J. Overbye, Cognitive Task Analysis of Electric Power System Control Center Operations, Human Factors and Ergonomics Society Annual Meeting Proceedings, Vol.52, No.24, pp.1934-1938 - September 2008.

[22] Ni, J.; Zhang, K.; Lin, X.; Shen, X. Securing Fog Computing for Internet of Things Applications: Challenges and Solutions. IEEE Communacition Surv. Tutor. 2017, 20, 601-628. 
BALKAN JOURNAL OF ELECTRICAL \& COMPUTER ENGINEERING， Vol. 10, No. 1, January 2022

[23] Miller, G. The cognitive revolution: a historical perspective, Trends in Cognitive Sciences, 2003, Vol.7, No.3, pp.141-144.

[24] Seker, S, Dikun, J. Physical Limits for Self-Contained Systems: An Example of the Human Brain as a Cognitive System. The Journal of Cognitive Systems, 2018, Vol.3, No.2, pp.28-29.

[25] Militello, L.G., Dominguez, C.O., Lintern, G., Klein, G. The Role of Cognitive Systems Engineering in the Systems Engineering Design Process, System Engineering, 2010, Vol.13, No.3, pp.261-273.

[26] Alafi, B . A New Cognitive System Modeling with the Combination of Expert and Neural Network Systems. The Journal of Cognitive Systems, 2018, Vol.3, No.2, pp.34-35.

\section{BIOGRAPHIES}

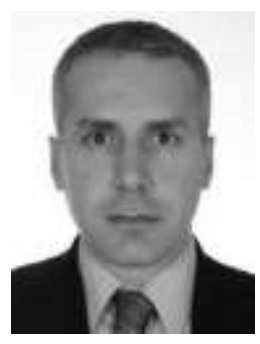

TAHIR CETIN AKINCI received bachelor's degree in electrical engineering in 2000. Dr. Akinci received Master's and $\mathrm{PhD}$ degrees from Istanbul Marmara University in 2005 and 2010, respectively. He works as a full professor in the Department of Electrical Engineering at Istanbul Technical University (ITU). At the same time, he works as a visiting scholar at the University of California Riverside (UCR) to do his postdoctoral research.

His research studies includes on Cognitive systems, Artificial intelligence methods, Machine learning, Electric power systems and data analytics.

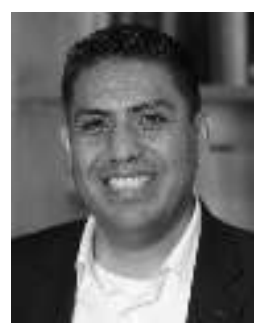

ALFREDO A. MARTINEZ-MORALES is the Managing Director of the Southern California Research Initiative for Solar Energy (SC-RISE) and Research Professor at the Bourns College of Engineering Center for Environmental Research and Technology (CE-CERT). Dr. Martinez-Morales received his Ph.D., M.S. and B.S. degrees in electrical engineering from University of California Riverside (UCR).

His current research includes solar cells, alkali metalion batteries, highly integrated renewables, energy storage systems, and microgrids. Dr. Martinez-Morales is a principal investigator in the Sustainable Integrated Grid Initiative (SIGI) at UCR, and has contributed in the engineering, permitting, and deployment of the SIGI smartgrid testbed system and multiple microgrids throughout Southern California. 